\title{
Long-term outcome of bronchoscopically resected endobronchial typical carcinoid tumors
}

Heyman Luckraz, FRCS, ${ }^{a}$ Khalid Amer, FRCS (CTh), ${ }^{a}$ Lynne Thomas, RGN, ${ }^{a}$ Allen Gibbs, FRCPath, ${ }^{\text {b }}$ and Eric G. Butchart, FRCS ${ }^{a}$
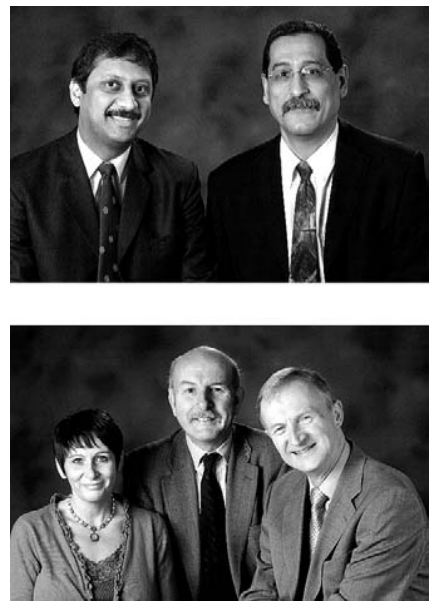

Drs Luckraz and Amer (top left to right); and Drs Thomas, Gibbs, and Butchart (bottom left to right)

Earn CME credits at http:// cme.ctsnetjournals.org
From the Cardiothoracic Unit ${ }^{\mathrm{a}}$ and Pathology Department, ${ }^{\mathrm{b}}$ University Hospital of Wales, Cardiff, United Kingdom.

Received for publication Jan 10, 2006; revisions received Jan 30, 2006

Address for reprints: Heyman Luckraz, FRCS, Cardiothoracic Unit, University Hospital of Wales, Heath Park, Cardiff CF4 4XW, United Kingdom (E-mail: HeymanLuckraz@aol.com).

J Thorac Cardiovasc Surg 2006;132:113-5

$0022-5223 / \$ 32.00$

Copyright (C) 2006 by The American Association for Thoracic Surgery

doi:10.1016/j.jtcvs.2006.01.061
Background: Typical pulmonary carcinoid tumors represent less than $1 \%$ of lung tumors. In a subgroup of patients with this abnormality, the tumor is entirely endobronchial. We assessed the long-term outcome of such cases in which the patient was managed with endobronchial resection only.

Methods: Patients who underwent bronchoscopic resection for a typical carcinoid tumor were identified through case records and histology reports. Data were collected retrospectively, but follow-up was on a prospective basis through the outpatient clinic.

Results: Between 1978 and 2004, 28 patients underwent bronchoscopic resection of endobronchial carcinoid tumors. The mean age was 49 years (standard deviation, 19 years; age range, $11-82$ years), with $46 \%$ (13/28) of the patients being male. The tumor arose from the left bronchial tree in $61 \%$ (17/28), with the most common site being the left lower lobe bronchus (8/28). On average, patients required 5 bronchoscopic resections to achieve complete resection. The median follow-up was 8.8 years (interquartile range, 4.5-13.7 years). At 1 and 10 years, $100 \%$ and 94\% of patients were disease free, respectively. The 1- and 10-year survivals were $89 \%$ (interquartile range, $84 \%-93 \%$ ) and $84 \%$ (interquartile range, $77 \%-91 \%$ ), respectively.

Conclusion: In a selected group of patients, proximal polypoid typical bronchial carcinoid tumors can be treated endobronchially with good outcome.

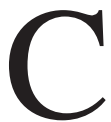

arcinoid tumors arise from the neuroendocrine cells within the mucosal layer of the tracheobronchial tree. Typical carcinoid tumors represent the relatively benign end of the spectrum. Initially classified as bronchial adenoma, carcinoid tumor was described as a separate entity by Hamperl ${ }^{1}$ in 1937 . The majority (90\%) of these tumors arise in the walls of medium-to-large airways. ${ }^{2}$ Characteristically, part of the lesion protrudes as a domed mass into the lumen, with the remainder of the tumor extending into the wall of the airway and the adjacent lung, giving them a dumbbell appearance. However, a small proportion of carcinoid tumors are entirely endobronchial, forming a polyp. ${ }^{3}$ Endobronchial typical carcinoid tumors usually present with recurrent chest infections, wheezing, hemoptysis, and very rarely with symptoms of carcinoid syndrome (flushing, diarrhea, wheeze, abdominal pain, loss of appetite, and weight loss). Many are labeled as "asthma" before bronchoscopy. Hilar metastases are rare (6\%), with distant metastases (liver, lung, brain, adrenal, and bone) even rarer. ${ }^{4}$ We reviewed our experience over a 26-year period with the management of endobronchial, proximal, polypoid, typical carcinoid lung tumors using bronchoscopic resection and report the long-term outcome.

\section{Methods}

Patients who underwent bronchoscopic resection for typical carcinoid tumors were identified through case records and histology reports. Data were collected retrospectively, but follow-up was on a prospective basis through the outpatient clinic. 


\section{Abbreviations and Acronyms \\ $\mathrm{CT}=$ computed tomography \\ $\mathrm{IQR}=$ interquartile range}

Patient selection for bronchoscopic resection was carried out by using both preoperative and perioperative parameters. Preoperative parameters included computed tomographic (CT) scans showing no signs of bronchial wall infiltration (bronchial wall irregularities, bronchial wall thickening, and extrabronchial tumor) or enlarged lymph nodes. Operative criteria included a proximally based polypoid tumor with a narrow point of attachment, which was confirmed to be a typical carcinoid tumor (without mitoses) on histologic analysis.

The bronchoscopic resection was carried out after achievement of general anesthesia and full muscle relaxation. The rigid bronchoscope with side-port continuous mechanical ventilation was used. The polyp was removed piecemeal with biopsy forceps, as permitted by visibility. Diathermy was used to control hemorrhage if necessary but was rarely required. This procedure was repeated fortnightly to achieve complete excision of the whole polyp. The latter was confirmed on the basis of 2 successive negative bronchoscopies both on macroscopic and microscopic grounds. Thereafter patients underwent a 6-month check-up bronchoscopy and annual visits to the outpatient department to assess their condition and progress. Patients' follow-up after achievement of microscopic clearance included the bronchoscopic check-up at 6 months, and if still clear, then patients were solely reviewed in the outpatient department. During this visit, a targeted medical history was taken, and relevant clinical examination was carried out. A chest radiograph was also taken. If there were concerns on either the history, examination, or chest radiograph, then a CT scan was performed with octreotide labeling if deemed necessary.

Recurrence was defined as either macroscopic or microscopic evidence of tumor after 2 consecutive negative check-up bronchoscopies.

\section{Results}

Between 1978 and 2004, 28 patients underwent bronchoscopic resection for typical polypoid carcinoid tumor. The mean age was 49 years (standard deviation, 19 years; age range, $11-82$ years), with $46 \%(13 / 28)$ of the patients being male.

Recurrent chest infection was the presenting feature in $43 \%$ of patients. Hemoptysis was reported by $57 \%$. Other symptoms included persistent cough $(63 \%)$, breathlessness (45\%), wheeze (45\%), and chest pain (18\%).

The tumor arose from the left bronchial tree in $61 \%$ $(17 / 28)$, with the most common site being the left lower lobe bronchus (29\%). The locations of the remaining tumors were as follows: right main bronchus (3\%), right upper lobe bronchus $(11 \%)$, bronchus intermedius $(14 \%)$, right lower lobe bronchus (11\%), left main bronchus (25\%), and left upper lobe bronchus (7\%). All of the tumors originated from the cartilaginous wall of the bronchus.
On average, patients required 5 bronchoscopic resections to achieve complete resection. Significant hemorrhage was experienced in only 1 patient, whose symptoms were managed by applying local pressure with an epinephrine-soaked Lahey swab mounted on a long forceps.

The median follow-up was 8.8 years (interquartile range [IQR], 4.5-13.7 years). At 1 and 10 years, $100 \%$ and $94 \%$ of patients were disease free, respectively. One patient had recurrence of the tumor at 80 months from initial clearance and underwent successful treatment by means of lobectomy.

The 1- and 10-year survivals were $89 \%$ (IQR, 84\%-93\%) and $84 \%$ (IQR, 77\%-91\%), respectively. Cause of death was not related to the diagnosis of carcinoid tumor and included adenocarcinoma (2 patients), ischemic heart disease, and biliary sepsis.

\section{Discussion}

The classification of carcinoid tumors stems from the recommendations of the World Health Organization. ${ }^{5}$ These recommendations proposed the labeling of the benign end of the spectrum as "typical," whereas "atypical carcinoid" was used if the mitotic rate was up to 10 per high-power field. ${ }^{6}$ However, there is still controversy concerning the optimal management of typical bronchial carcinoid tumors.

Parenchyma-saving procedures were not favored in the 1980s after the report by Aberg and colleagues, ${ }^{7}$ who advocated that prognosis is adversely affected, especially after 7 years, in patients who had undergone bronchoplastic procedures. However, more recently, there have been favorable reports with good long-term outcomes in patients who have undergone bronchotomy and excision of carcinoid tumors. ${ }^{8}$

Proponents of radical excision (lobectomy and pneumonectomy) promote the theory that the intraluminal extension of carcinoid tumor represents the tip of the iceberg. Although this is true in a significant number of patients with carcinoid tumors, there exists a subpopulation of patients in whom the entire tumor is endobronchial, forming a polypoid lesion. ${ }^{3}$

A review of endobronchial resection in 1995 confirmed that the bronchoscopic approach represented a successful modality of treatment. ${ }^{9}$ The authors reported that after single bronchoscopic resection, they proceeded to lung resection in 6 of 11 patients because they could not rule out residual tumor. Interestingly, the histology of the resected specimen excluded any residual tumor. This prompted the same group to perform a prospective study of endobronchial resection. In this series complete resection was achieved bronchoscopically in $74 \%$ (14/19) of patients. ${ }^{10}$ However, the failure to clear the tumor was entirely due to the inaccessibility of the tumor, which was in the subsegmental bronchi.

Previously reported modes of bronchoscopic resection for carcinoid tumor include the use of electrocautery and the 
ND:YAG laser. Our technique involved the use of biopsy forceps and removal of the tumor piecemeal. All of these techniques have proved to be effective in achieving complete resection.

Suitability for bronchoscopic resection should be fully assessed by using both preoperative and perioperative parameters, including high-resolution $\mathrm{CT}$ scanning to exclude bronchial wall and lymph node involvement as well as the presence of a proximally located polypoid tumor with confirmation of typical carcinoid on histology. Positron emission tomographic scanning might also be useful for evaluating doubtful lymphadenopathy. Endobronchial ultrasonographic evaluation was reported by Tremblay. ${ }^{11}$ His experience with endobronchial ultrasonography (the ultrasound probe being fed through the flexible bronchoscope) allowed a better assessment of the bronchial wall than with CT scanning.

\section{References}

1. Hamperl U. Uber gutarige bronchialtumoren (cylindrome und carcinoide). Virchows Arch Pathol Anat. 1937;300:46-88.
2. Gmelian JT, Bensch KG, Liebow AA. Cells of Kultchitsky type in bronchioles and their relation to the origin of peripheral cacinoid tumour. Lab Invest. 1967;17:88-98.

3. Addis BJ, Corrin B. Other epithelial tumours of the lung. In: Symmers WStC, editor. Systemic pathology: the lungs. 3rd ed. New York: Churchill Livingstone; 1990. p. 374.

4. Amgoni MG, Woolner LB, Bernatz PE. Atypical endocrine tumours of the lung. J Thorac Cardiovasc Surg. 1972;64:413-21.

5. World Health Organization Classification of Tumours. Pathology and genetics. tumours of the lung, pleura and heart. Lyon, France: IARC Press; 2004.

6. Gould VE, Linnoila RI, Memoli VA, Warren WH. Neuroendocrine components of the bronchopulmonary tract: hyperplasias, dysplasias and neoplasms. Lab Invest. 1983;49:519-37.

7. Aberg A, Blondal T, Nou E, Malmeus J. The choice of operation for bronchial carcinoids. Ann Thorac Surg. 1981;32:19-22.

8. Tastepe A, Kurul I, Demircan S, Liman S, Kaya S, Cetin G. Long-term survival following bronchotomy for polypoid bronchial carcinoid tumours. Eur J Cardiothorac Surg. 1998;14:575-7.

9. Sutedja G, Schreurs A, Vanderschueren R, Kwa B, van der Werff T, Postmus PE. Bronchoscopic therapy in patients with intraluminal typical bronchial carcinoid. Chest. 1995;107:556-8.

10. Van Boxem TJ, Venmans BJ, van Moulik JC, Postmus PE, Sutedja TG. Bronchoscopic treatment of intraluminal typical carcinoid: a pilot study. J Thorac Cardiovasc Surg. 1998;116:402-6.

11. Tremblay A. Endobronchial ultrasonography: extending the reach of the bronchoscope beyond the airway wall. CMAJ. 2003;169:586. 